\title{
Understandings of the Incremental Backstepping Control through Theoretical Analysis under the Model Uncertainties
}

\author{
Byoung-Ju Jeon ${ }^{1}$, Min-Guk Seo ${ }^{2}$, Hyo-Sang Shin $^{3}$ and Antonios Tsourdos ${ }^{4}$
}

\begin{abstract}
In this paper, theoretical analysis on the incremental backstepping control is suggested especially under the existence of model uncertainties. This algorithm is proposed in the previous studies by modifying the backstepping method to reduce model dependency. Because this method is a type of nonlinear control and the model uncertainties are assumed to be considered, it is difficult to have theoretical analysis, which causes lack of understandings about this algorithm. Therefore, this paper suggests closed-loop analysis with simplified dynamics under the model uncertainty. Transfer function is derived and poles, stability condition, steady state error, and settling time are presented. In addition, the effects of model uncertainties and gains are identified through analysis. Proposed analysis is meaningful in terms of establishing critical understandings about the algorithm, even though the simplified dynamics is applied for analysis purpose.
\end{abstract}

\section{INTRODUCTION}

Gain-scheduled linear feedback controller is the most widely applied control method, which uses a number of local linear controllers with scheduled gains to cover the whole flight envelope. There exists well-established linear control theory, so certification becomes easier thanks to the wellaccepted robustness and performance metrics. However, if there is flight envelope region with significant nonlinearities, accurate tracking cannot be guaranteed with linear control because it is based on linearized nominal aircraft model. Additionally, gain scheduling in traditional linear control framework requires enormous workload.

Nonlinear control algorithms, on the other hand, do not suffer from those kinds of drawbacks. Consequently, backstepping method [1], a recursive nonlinear control algorithm originally proposed to eliminate the relative-degree restriction of passive design, also has advantages from a standpoint mentioned before. It has been successfully applied in aerial systems for benchmark examples and real aerial platforms [2]- [7]. Nevertheless, backstepping method belongs to the model-based control strategy. It indicates that the method

\footnotetext{
*The authors would like to acknowledge that this research was supported by EU under a Horizon 2020 project, called INCEPTION (Incremental Nonlinear flight Control supplemented with Envelope ProtecTION techniques).

${ }^{1}$ Byoung-Ju Jeon is with School of Aerospace, Transport and Manufacturing (SATM), Cranfield University, College Road, Cranfield, United Kingdom B. Jeon@cranfield.ac.uk

${ }^{2}$ Min-Guk Seo is with School of Aerospace, Transport and Manufacturing (SATM), Cranfield University, College Road, Cranfield, United Kingdom Minguk. Seo@cranfield.ac.uk

${ }^{3}$ Hyo-Sang Shin is with School of Aerospace, Transport and Manufacturing (SATM), Cranfield University, College Road, Cranfield, United Kingdom h.shinecranfield.ac.uk

${ }^{4}$ Antonios Tsourdos is with School of Aerospace, Transport and Manufacturing (SATM), Cranfield University, College Road, Cranfield, United Kingdom a.tsourdos@cranfield.ac.uk
}

is sensitive to model uncertainties like aerodynamic uncertainties. However, it is difficult to get accurate model which requires tremendous amount of cost. Hence, the incremental backstepping(IBKS) control [8] [9] is suggested to reduce the dependency on the aircraft model, which is known to require accurate knowledge only about the control effectiveness.

This paper suggests theoretical analysis for IBKS method under the model uncertainties. This method is a type of nonlinear control, so it has shortcomings in theoretically well established analysis. Absolute stability using Lyapunov candidate function is almost the only metric for nonlinear control that is commonly discussed so far. It is hard to tell relative stability or fundamental performance metrics analytically. Furthermore, analysis becomes more complicated if model uncertainties are introduced, since nonlinearities can not be completely cancelled for the closed-loop system. Besides, it is difficult to find previous studies which successfully perform theoretical analysis for IBKS under the model uncertainties. There only exist papers [8] - [10] and [11] which show closed loop characteristics under the model uncertainties just through simulations or experiments.

In this paper, IBKS is derived in Sec. III and closed loop analysis is performed in Sec. IV, with short period mode dynamics suggested in Sec. II. Simplified dynamics is applied for analysis purpose, and model uncertainties are considered in closed loop analysis. Proposed analysis is meaningful in terms of establishing critical understandings about the closed loop system with IBKS. Transfer function is calculated and consequently, poles, stability condition, steady state error, and settling time are presented to discuss more about the stability and the performance metric. Several properties like the effects of model uncertainties and gains on the system characteristic can be understood. Through some case studies, these features are more easily explained and finally shown in the simulation.

\section{PRELIMINARIES : DYNAMICS}

Short period mode dynamics, one of the longitudinal oscillation modes with high natural frequency, is applied for the derivation of IBKS and its closed loop analysis. This mode is of paramount importance in the flight control, because one of the main purposes of the stability augmentation system for the airplane is to improve characteristics about this mode and it is highly related to the angle of attack control.

$$
\begin{aligned}
\dot{\alpha} & =Z_{\alpha}(M, \alpha) \alpha+q+Z_{\delta}(M, \alpha) \delta \\
\dot{q} & =M_{\alpha}(M, \alpha) \alpha+M_{q}(M, \alpha) q+M_{\delta}(M, \alpha) \delta
\end{aligned}
$$


where the state variables $\alpha$ and $q$ represent the angle of attack and the pitch rate. The control input $\delta$ corresponds to the deflection of the elevator. $Z_{\alpha}, Z_{\delta}, M_{\alpha}, M_{q}$ and $M_{\delta}$ are aerodynamic derivatives.

This simplified dynamics is utilized for the simplicity of analysis which will be performed in Sec. IV with consideration of model uncertainties. It's still meaningful enough to have understandings about the effects of IBKS controller and the characteristics of the closed loop systems under the model uncertainties. As a future work, this study can be extended to the analysis with full 6-DoF dynamics.

\section{DERIVATION OF CONTROL LAWS}

IBKS controller is based on the backstepping method, which requires that the dynamics should be in the strictly feedback form. Therefore, this approximated dynamics (2) is applied for the control law derivation. In the original dynamics (1), $Z_{\delta} \delta$ which is related to non-minimum phase is neglected. It is often made assumption in flight control systems design. Additionally, aerodynamic derivatives estimates $(\hat{.})$ identified from wind turnel test or aeroprediction are used, and they are handled in piece-wise way in order to easily utilize existing analysis framework for the linear time-invariant system in Sec. IV.

$$
\begin{aligned}
\dot{\alpha} & =\hat{Z}_{\alpha} \alpha+q \\
\dot{q} & =\hat{M}_{\alpha} \alpha+\hat{M}_{q} q+\hat{M}_{\delta} \delta
\end{aligned}
$$

State errors can be defined as below.

$$
\begin{aligned}
& z_{1}=\alpha-\alpha_{c} \\
& z_{2}=q-q_{c}
\end{aligned}
$$

where subscript $c$ indicates command.

Asymptotic stability for nonlinear system can be guaranteed if Lyapunov candidate function becomes positive deflinite and its derivative becomes negative definite. To derive IBKS control command which satisfies asymptotic stability, 2 cascaded steps are performed.

First, Lyapunov candidate function $V_{1}$ with only consideration of $z_{1}$ is selected as

$$
V_{1}=\frac{1}{2} z_{1}^{2}
$$

which is positive definite.

Then, its derivative becomes

$$
\begin{aligned}
\dot{V}_{1} & =z_{1} \dot{z}_{1} \\
& =z_{1}\left(\dot{\alpha}-\dot{\alpha}_{c}\right) \\
& =z_{1}\left(\hat{Z}_{\alpha} \alpha+q-\dot{\alpha}_{c}\right)
\end{aligned}
$$

In order to satisfy Lyapunov stability condition, psedocommand $q_{c}$ is derived as

$$
q_{c} \triangleq-C_{1} z_{1}-\hat{Z}_{\alpha} \alpha+\dot{\alpha}_{c}
$$

which makes negative definite $\dot{V}_{1}=-C_{1} z_{1}^{2}$.

The first step is related to the outer-loop control design which just classical backstepping design procedure is applied to. For the inner-loop control design, $q$ dynamics in (2) is modified with the assumption that the states $\alpha, q$ and the control input $\delta$ can be expressed as a combination of trim values $(\cdot)_{0}$ and disturbances $\Delta(\cdot)$ around them. This assumption is valid especially for the environment with the sufficiently high sampling rate.

$$
\begin{aligned}
\dot{q} & =\hat{M}_{\alpha}\left(\alpha_{0}+\Delta \alpha\right)+\hat{M}_{q}\left(q_{0}+\Delta q\right)+\hat{M}_{\delta}\left(\delta_{0}+\Delta \delta\right) \\
& =\dot{q}_{0}+\hat{M}_{\alpha} \Delta \alpha+\hat{M}_{q} \Delta q+\hat{M}_{\delta} \Delta \delta
\end{aligned}
$$

$\Delta \alpha$ and $\Delta q$, the increments in states, can be ignored because the effects of them are much smaller than the one with increment in input, $\Delta u$, which directly affects the pitch moment. Then, the $q$ dynamics for the design of IBKS control becomes

$$
\dot{q} \simeq \dot{q}_{0}+\hat{M}_{\delta} \Delta \delta
$$

As the second step, Lyapunov candidate function $V_{2}$ with consideration of both $z_{1}$ and $z_{2}$ is selected as

$$
V_{2}=\frac{1}{2} z_{1}^{2}+\frac{1}{2} z_{2}^{2}
$$

which is positive definite.

Then, its derivative becomes

$$
\begin{aligned}
\dot{V}_{2} & =z_{1} \dot{z}_{1}+z_{2} \dot{z}_{2} \\
& =z_{1}\left(\hat{Z}_{\alpha} \alpha+q-\dot{\alpha}_{c}\right)+z_{2}\left(\dot{q}_{0}+\hat{M}_{\delta} \Delta \delta-\dot{q}_{c}\right)
\end{aligned}
$$

By using pseudo-command equation which is suggested in (6), $\dot{V}_{2}$ becomes

$$
\dot{V}_{2}=z_{1}\left(-C_{1} z_{1}+z_{2}\right)+z_{2}\left(\dot{q}_{0}+\hat{M}_{\delta} \Delta \delta-\dot{q}_{c}\right)
$$

In order to satisfy Lyapunov stability condition, the control command $\Delta \delta$ is derived as

$$
\Delta \delta \triangleq \frac{1}{\hat{M}_{\delta}}\left(-C_{2} z_{2}+\dot{q}_{c}-z_{1}-\dot{q}_{0}\right)
$$

which makes negative definite $\dot{V}_{2}=-C_{1} z_{1}^{2}-C_{2} z_{2}^{2}$.

Therefore, the final form of the derived IBKS control is

$$
\begin{aligned}
q_{c} & =-C_{1} z_{1}-\hat{Z}_{\alpha} \alpha+\dot{\alpha}_{c} \\
\delta & =\delta_{0}+\Delta \delta \\
& =\frac{1}{\hat{M}_{\delta}}\left(-C_{2} z_{2}+\dot{q}_{c}-z_{1}-\dot{q}_{0}\right)+\delta_{0}
\end{aligned}
$$

To make the angle of attack $\alpha$ go to the desired value $\alpha_{c}$, pseudo-command $q_{c}$ is derived and it is achieved that $q$ goes to $q_{c}$ by the actual control input $\delta$. Only information about $\hat{Z}_{\alpha}$ and $\hat{M}_{\delta}$ is required to formulate the control law. It's because the effects of the aerodynamic derivatives $\hat{M}_{\alpha}$ and $\hat{M}_{q}$ are disappeared by neglecting $\Delta \alpha$ and $\Delta q$ in $q$ dynamics. Model dependency is reduced, and there is no need to worry about the uncertainties in $\hat{M}_{\alpha}$ and $\hat{M}_{q}$. Instead, additional measurements $\delta_{0}$ and $\dot{q}_{0}$ related to current control surface deflection and state derivatives are required for control law implementation. 


\section{Closed-LOOP ANLAYSis}

In this chapter, closed-loop analysis is performed. The control input (13) derived in the previous section is substituted to the original dynamics (1) with $Z_{\delta}=0$ condition and with the other true aerodynamic derivatives in piece-wise way. $Z_{\delta}$ can be usually neglected for the large airplanes, even though it is hard to ignore $Z_{\delta}$, which has always negative sign, for the air vehicles with high maneuverability like missiles. For analysis, transfer function for the closed-loop system is derived and then, the equation for the steady state error as one of performance metrics is suggested.

To easily provide more interpretations and understandings about the control algorithm, case studies are performed when there exists uncertainty respectively in $\hat{Z}_{\alpha}$ and $\hat{M}_{\delta}$. For each case, transfer function is given and consequently, poles, stability condition, steady state error, and settling time are suggested to discuss more about the stability and the performance metric. In addition, several properties like the effects of model uncertainties and gains can be understood through analysis.

\section{A. General analysis}

Before analysis, it is good to decide how to deal with current state derivative and control surface deflection measurements $\dot{q}_{0}$ and $\delta_{0}$. In this paper, they are suggested as following.

$$
\begin{aligned}
& \dot{q}_{0}=M_{\alpha} \alpha+M_{q} q+M_{\delta} \delta_{0} \\
& \delta_{0}=\delta(t-\tau)
\end{aligned}
$$

Current state derivative measurement $\dot{q}_{0}$ is modelled by using piece-wise version of (1). If an actuator is assumed to be perfect, control surface deflection becomes the same with generated control command. Then, current control surface deflection measurement $\delta_{0}$ can be regarded as control command generated in the previous step. $\tau$ stands for step size.

Under the assumption of constant $\alpha_{c}$, i.e. $\dot{\alpha}_{c}=\ddot{\alpha}_{c}=0$, transfer function can be derived as below.

where

$$
\frac{\alpha(s)}{\alpha_{c}(s)}=\frac{T(s)}{s^{2}+2 \zeta \omega_{n} s+\omega_{n}^{2}}
$$

$$
\begin{aligned}
T(s) & =\left(C_{1} C_{2}+1\right) \frac{M_{\delta}}{\phi(s)} \\
2 \zeta \omega_{n} & =-\left\{Z_{\alpha}+M_{q}-\frac{M_{\delta}}{\phi(s)}\left(C_{1}+C_{2}+M_{q}+\hat{Z}_{\alpha}\right)\right\} \\
\omega_{n}^{2}= & \left(1-\frac{M_{\delta}}{\phi(s)}\right)\left(Z_{\alpha} M_{q}-M_{\alpha}\right) \\
& +\frac{M_{\delta}}{\phi(s)}\left\{C_{2}\left(\hat{Z}_{\alpha}-Z_{\alpha}\right)+\left(C_{1} C_{2}+1\right)\right\}
\end{aligned}
$$

$\phi(s)$ is written as below.

$$
\phi(s)=\hat{M}_{\delta}\left(1-e^{-\tau s}\right)+M_{\delta} e^{-\tau s}
$$

For step input, $\alpha_{c}(s)=\frac{K}{s}$. Therefore, $\alpha(s)$ becomes

$$
\alpha(s)=K \frac{T(s)}{s^{2}+2 \zeta \omega_{n} s+\omega_{n}^{2}} \frac{1}{s}
$$

Steady state error can be derived from

$$
e_{s s}=\alpha_{c}-\lim _{t \rightarrow \infty} \alpha(t)=\alpha_{c}-\lim _{s \rightarrow 0} s \alpha(s)
$$

Then, the steady state error $e_{s s, 1}$ with IBKS controller can be represented as below.

$$
e_{s s}=K\left\{\frac{\eta_{2}}{\eta_{1}+\eta_{2}}\right\}
$$

$\eta_{1}$ and $\eta_{2}$ in steady state error equation can be written as below.

$$
\begin{aligned}
& \eta_{1}=C_{1} C_{2}+1 \\
& \eta_{2}=C_{2}\left(\hat{Z}_{\alpha}-Z_{\alpha}\right)
\end{aligned}
$$

\section{B. Case study}

1) Case with only uncertainty in $\hat{Z}_{\alpha}$ : If there exists uncertainty only in $\hat{Z}_{\alpha}=Z_{\alpha}(1+\Delta)$ and uncertainty in $\hat{M}_{\delta}$ is neglected(i.e. $\hat{M}_{\delta}=M_{\delta}$ ), then $\left.\phi(s)\right|_{\hat{M}_{\delta}=M_{\delta}}=M_{\delta}$.

Therefore, transfer function becomes

$$
\frac{\alpha(s)}{\alpha_{c}(s)}=\frac{T(s)}{s^{2}+2 \zeta \omega_{n} s+\omega_{n}^{2}}
$$

where

$$
\begin{aligned}
T(s) & =\left(C_{1} C_{2}+1\right) \\
2 \zeta \omega_{n} & =\left(C_{1}+C_{2}\right)+Z_{\alpha} \Delta \\
\omega_{n}^{2} & =\left(C_{1} C_{2}+1\right)+C_{2} Z_{\alpha} \Delta
\end{aligned}
$$

Then the poles $p_{1}$ can be suggested as below.

$$
p=\frac{-2 \zeta \omega_{n} \pm \sqrt{\left(2 \zeta \omega_{n}\right)^{2}-4 \omega_{n}^{2}}}{2}
$$

It is observed from the above equation that the poles move to more left side as gains increase, which means relative stability can be improved with high gains.

The condition to maintain stability with model uncertainty can be proposed from $2 \zeta \omega_{n}>0$ (Cond.1) under $\omega_{n}^{2}>0$ (Cond.2) as follows.

$$
\mathscr{G}=\left\{C_{1}, C_{2} \in \mathbb{R}_{>0} \mid \text { Cond. } 1 \& \text { Cond. } 2\right\}
$$

Cond. $1: C_{1}+C_{2}>-Z_{\alpha} \Delta$

Cond. 2 : $C_{1} C_{2}+C_{2} Z_{\alpha} \Delta>-1$

Settling time, when the magnitude of the error is reduced within $5 \%$, can be calculated by using approximated equation below

$$
t_{s}= \begin{cases}\frac{3.2}{\zeta \omega_{n}} & \text { if } 0<\zeta<0.69 \\ \frac{4.5}{\omega_{n}} \zeta & \text { if } \zeta>0.69\end{cases}
$$

where

$$
\begin{aligned}
\omega_{n} & =\sqrt{\left(C_{1} C_{2}+1\right)+C_{2} Z_{\alpha} \Delta} \\
\zeta & =\frac{C_{1}+C_{2}+Z_{\alpha} \Delta}{2 \sqrt{\left(C_{1} C_{2}+1\right)+C_{2} Z_{\alpha} \Delta}}
\end{aligned}
$$

$\zeta$ and $\omega_{n}$ represent damping ratio and natural frequency. 
The steady state error $e_{s s}$ becomes

$$
e_{s s}=K \frac{C_{2} Z_{\alpha} \Delta}{\left(C_{1} C_{2}+1\right)+C_{2} Z_{\alpha} \Delta}
$$

From derived equation above, following properties related to the steady state error can be found. First, $\left|e_{s s}\right|$ gets larger as $|\Delta|$ increases. Second, $e_{s s}$ can be reduced with high gains.

2) Case with only uncertainty in $\hat{M}_{\delta}$ : If there exists uncertainty only in $\hat{M}_{\delta}=M_{\delta}(1+\Delta)$ and uncertainty in $\hat{Z}_{\alpha}$ is neglected(i.e. $\hat{Z}_{\alpha}=Z_{\alpha}$ ), analysis can be performed as following.

If $\tau$ is small enough, $e^{-\tau s} \simeq 1$ and $\left.\phi(s)\right|_{\hat{z}_{\alpha}=z_{\alpha}} \simeq M_{\delta}$. Therefore, transfer function becomes

$$
\frac{\alpha(s)}{\alpha_{c}(s)}=\frac{\left(C_{1} C_{2}+1\right)}{s^{2}+\left(C_{1}+C_{2}\right) s+\left(C_{1} C_{2}+1\right)}
$$

Then the poles $p_{1}$ can be suggested as below.

$$
p=\frac{-\left(C_{1}+C_{2}\right) \pm \sqrt{\left(C_{1}-C_{2}\right)^{2}-4}}{2}
$$

From the above equation, following characteristics can be shown. First, poles can be expressed as a function of gains only, without any model parameters like aerodynamic derivatives. Second, when the gains are just positive, the poles are always located in the left half plane, which means the system always becomes stable. Third, poles go to more left side as gains become larger, which means relative stability can be enhanced with high gains.

Settling time can be calculated by using (24) with

$$
\begin{gathered}
\omega_{n}=\sqrt{C_{1} C_{2}+1} \\
\zeta=\frac{C_{1}+C_{2}}{2 \sqrt{C_{1} C_{2}+1}}
\end{gathered}
$$

Performance metrics related to fast response like settling time and rise time are also determined only by gain values.

The steady state error $e_{s s}$ always becomes zero because $\left.\eta_{2}\right|_{\hat{z}_{\alpha}=z_{\alpha}}=0$.

Under the condition that $\tau$ is small enough(i.e. the calculated control command is transmitted and reflected fast enough to the real control surface deflection), the system becomes robust with respect to the uncertinty in $\hat{M}_{\delta}$ even if this information is still required to implement IBKS. Ths is the one of the most important findings obtained from the theoretical analysis.

\section{Simulation}

Under the $Z_{\delta}=0$ condition, simulations are performed for each case with uncertainty in $\hat{Z}_{\alpha}$ and $\hat{M}_{\delta}$ respectively. Simulations for these 2 cases are carried out with specific values in table 1. For aerodynamic derivatives, piece-wise approach is considered. Several points were simulated, and the result from the case with $Z_{\alpha}=-0.0075, Z_{\delta}=0, M_{\alpha}=$ 1.4049, $M_{q}=-1.19$ and $M_{\delta}=-11.56$ is suggested in this paper as an example.
TABLE I

SimUlation PARAMETERS

\begin{tabular}{|c||c|}
\hline Parameter & Value \\
\hline \hline$\alpha_{c}$ & $2^{\circ}$ \\
\hline$C_{1}, C_{2}$ & 2 \\
\hline$\tau$ & 0.01 sec \\
\hline$\Delta$ & {$[-0.75,-0.5,-0.25,0,1,2,3,4]$} \\
\hline
\end{tabular}

A. Simulation results with only uncertainty in $\hat{Z}_{\alpha}$

Predicted $t_{s}, \omega_{n}, \zeta$ and $e_{s s}$ from the previous analysis are given in table 2. Fig.1 and Fig.2. suggest simulation results.

TABLE II

PREDicted RESULTS With $\Delta$ IN $\hat{Z}_{\alpha}$

\begin{tabular}{|c||c|c|c|c|}
\hline$\Delta$ & $e_{s s, 1}$ & $\omega_{n}$ & $\zeta$ & $t_{s}$ \\
\hline \hline-0.75 & 0.0045 & 2.2386 & 0.8947 & 1.7985 \\
\hline-0.5 & 0.0030 & 2.2377 & 0.8946 & 1.7990 \\
\hline-0.25 & 0.0015 & 2.2369 & 0.8945 & 1.7995 \\
\hline 0 & 0 & 2.2361 & 0.8944 & 1.8 \\
\hline 1 & -0.0060 & 2.2327 & 0.8941 & 1.8020 \\
\hline 2 & -0.0121 & 2.2293 & 0.8938 & 1.8041 \\
\hline 3 & -0.0182 & 2.2260 & 0.8934 & 1.8061 \\
\hline 4 & -0.0243 & 2.2226 & 0.8931 & 1.8082 \\
\hline
\end{tabular}

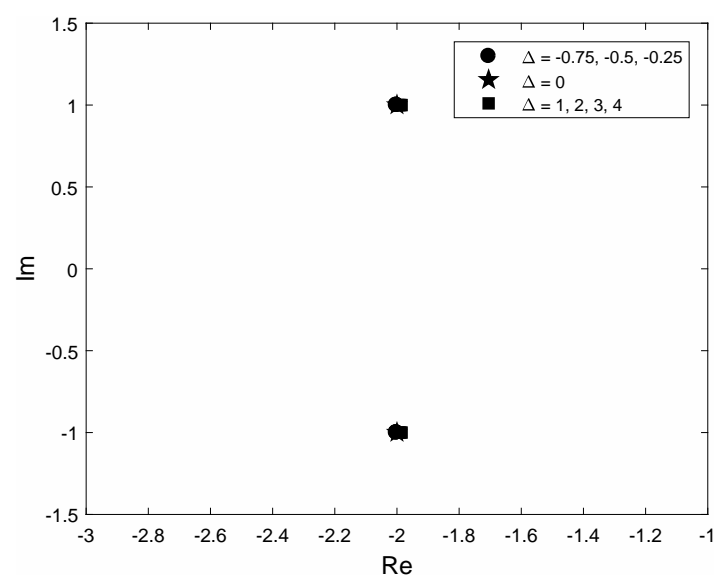

Fig. 1. Pole Trajectory ( $Z_{\alpha}$ Disturbance, IBKS)

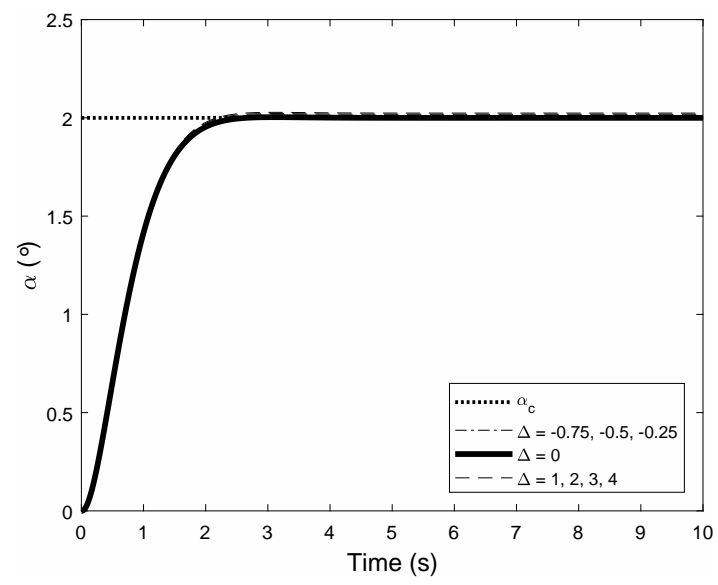

Fig. 2. Time Response ( $Z_{\alpha}$ Disturbance, IBKS) 
As shown in Fig. 1, uncertainty in $\hat{Z}_{\alpha}$ doesn't have much influence on the location of poles. Therefore, $\omega_{n}, \zeta$ and consequently $t_{s}$ do not change a lot, which is observed in Fig 2. This also happens in $\left|e_{s s}\right|$. Gains rather have more effects on the characteristics of the closed-loop system; in this example, gains are big enough to make poles located in left half plane, so the system becomes stable with every $\Delta$.

The predicted values summarized in table 2 have the same tendancy with the simulation results. $\left|e_{s s}\right|$ slightly increases as $|\Delta|$ goes up. As $\Delta$ becomes larger, $\omega_{n}$ and $\zeta$ decrease in a small amount, and $t_{s}$ increases a liittle bit. However, there's no big difference in performance metrics caused by the uncertainty $\Delta$ in $\hat{Z}_{\alpha}$

\section{B. Simulation results with only uncertainty in $\hat{M}_{\delta}$}

Predicted $t_{s}, \omega_{n}, \zeta$ and $e_{s s, 1}$ from the previous analysis are summarized in table 3 , and simulation results are suggested in Fig. 3 and Fig.4.

TABLE III

Predicted RESUlts With $\Delta$ IN $\hat{M}_{\delta}$

\begin{tabular}{|c||c|c|c|c|}
\hline$\Delta$ & $e_{s s, 1}$ & $\omega_{n}$ & $\zeta$ & $t_{s}$ \\
\hline \hline For all $\Delta$ & 0 & 2.2361 & 0.8944 & 1.8 \\
\hline
\end{tabular}

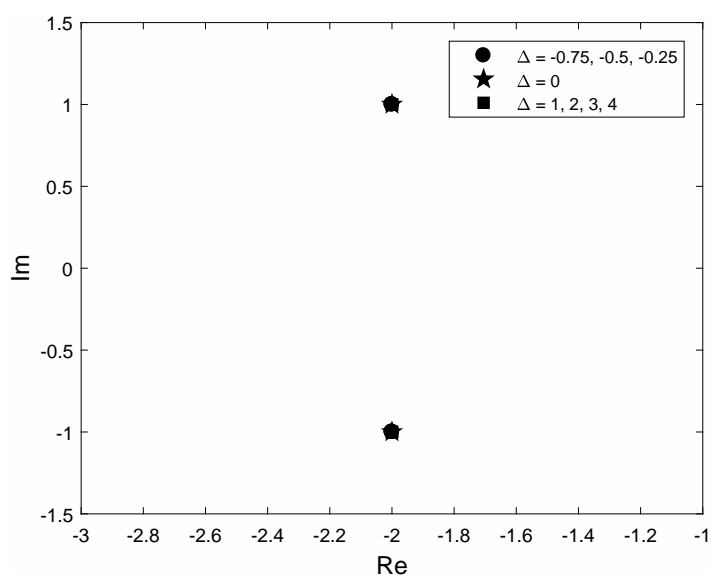

Fig. 3. Pole Trajectory ( $M_{\delta}$ Disturbance, IBKS)

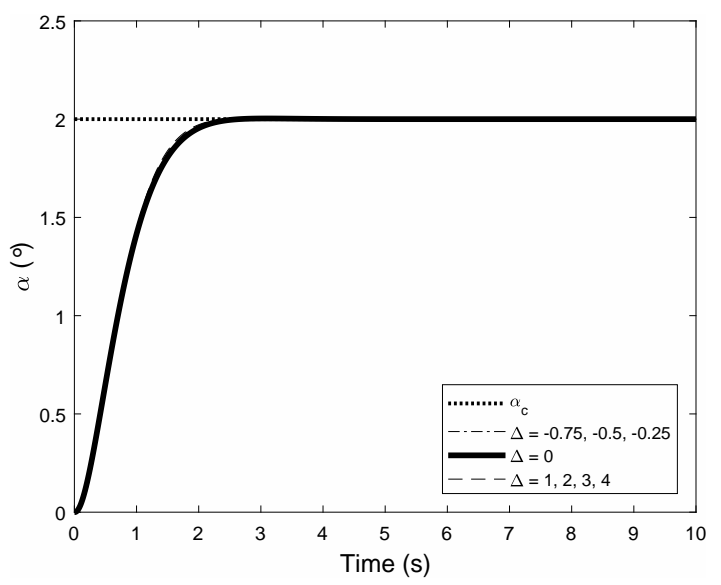

Fig. 4. Time Response ( $M_{\delta}$ Disturbance, IBKS)
Previous analysis indicates that $p, \omega_{n}, \zeta$ and $t_{s}$ are functions of gains only, and $e_{s s}$ is always zero. Therefore, table 3 suggests a result regardless of $\Delta$. Simulation results coincide with the prediction. It shows robust result with respect to $\Delta$ in $\hat{M}_{\delta}$. With positive gains, poles are always in the left half plane, so the system is stable all the time. There's no change in the location of poles. Conseqently, $\omega_{n}$, $\zeta$ and $t_{s}$ remain the same. $e_{s s}$ is always zero.

\section{CONCLUSIONS}

This paper proposes theoretical analysis with IBKS under the model uncertainties. IBKS algorithm is derived especially for the inner-loop control design. It is based on the backstepping method, but $\Delta \alpha$ and $\Delta q$ are ignored under the assumption of sufficiently high sampling rate. Consequently, the effects of the aerodynamic derivatives $\hat{M}_{\alpha}$ and $\hat{M}_{q}$ are disappeared and accurate knowledge only about the control effectiveness is required. In this paper, closed loop analysis is performed considering model uncertainties. Transfer function and steady state error are derived for general analysis. Case studies are performed when there exists uncertainty respectively in $\hat{Z}_{\alpha}$ and $\hat{M}_{\delta}$, to easily provide more interpretations and understandings about the control algorithm. For each case, transfer function is given and consequently, poles, stability condition, steady state error, and settling time are suggested to discuss further about the stability and the performance metric. For analysis purpose, simplified dynamics is used. Simulations are performed to check if expected characteristics from the analysis really appear.

Proposed analysis helps to establish critical understandings like the effects of model uncertainties and gains on the system characteristic. Identified properties from the analysis can be suggested as follows. If the uncertainty only exists in $\hat{M}_{\delta}$ and calculated control command is transmitted and reflected fast enough to the real control surface deflection, poles and the settling time can be expressed as a function of gains only, without any model parameters like aerodynamic derivatives. If the gains are just positive, poles are always located in the left half plane and the system is stable regardless of the uncertainty. Besides, $e_{s s, 1}$ always becomes zero. It indicates that the uncertinty in $\hat{M}_{\delta}$ doesn't affect stability and performance of the closed loop system, even if this information is still required to implement IBKS. This is the one of the most important findings obtained from the theoretical analysis. For the case with only uncertainty in $\hat{Z}_{\alpha},\left|e_{s s, 1}\right|$ increases as $|\Delta|$ increases and it can be reduced with high gains. For both cases, poles go to more left side as gains become larger, which means relative stability can be enhanced with high gains.

\section{ACKNOWLEDGMENT}

The authors would like to acknowledge that this research was supported by EU under a Horizon 2020 project, called INCEPTION (Incremental Nonlinear flight Control supplemented with Envelope ProtecTION techniques 


\section{REFERENCES}

[1] P. V Kokotovie, The Joy of Feedback: Nonlinear and Adaptive, IEEE Control Syst., pp. 717, 1992.

[2] J. R. Azinheira and A. Moutinho, A backstepping controller for path-tracking of an underactuated autonomous airship, Int. J. Robust Nonlinear Control, vol. 19, pp. 418441, 2009.

[3] J. R. Azinheira and A. Moutinho, Hover control of an UAV with backstepping design including input saturations, IEEE Trans. Control Syst. Technol., vol. 16, no. 3, pp. 517526, 2008.

[4] L. Zhong, L. Xiao-Geng, C. Bing-Gang, and C. Jun-Yi, An Optimal Backstepping Design for Blended Aero and Reaction-Jet Missile Autopilot, J. Appl. Sci., vol. 6, pp. 26232628, 2006.

[5] M. Seo, C. Lee, M. Tahk, J. Lee, and B. Jun, Design of Missile Autopilot using Approximate System and Backstepping Control, in AsiaPacific International Symposium on Aerospace Technology, 2012.

[6] F. Peter, F. Hellmundt, F. Holzapfel, and F. Chew, Anti-Windup Command Filtered Adaptive Backstepping Autopilot Design for a TailControlled Air-Defense Missile, in AIAA Guidance, Navigation, and Control conference, 2013, pp. 119.

[7] T. Lee and Y. Kim, "Nonlinear Adaptive Flight Control Using Backstepping and Neural Networks Controller", Journal of Guidance, Control, and Dynamics, Vol. 24, No. 4 (2001), pp. 675-682.

[8] P. Van Gils, E. van Kampen, C. C. de Visser, and Q. P. Chu, Adaptive Incremental Backstepping Flight Control for a High-Performance Aircraft with Uncertainties, AIAA SciTech Forum, no. January, pp. 126, 2016.

[9] A. Ait Haddou Ali, Q. P. Chu, E.-J. Van Kampen, and C. C. de Visser, Exploring Adaptive Incremental Backstepping using Immersion and Invariance for an F-16 aircraft, AIAA Guid. Navig. Control Conf., no. January, 2014.

[10] P. Acquatella, E.-J. van Kampen, and Q. P. Chu, Incremental Backstepping for Robust Nonlinear Flight Control, CEAS Conf. Guid. Navig. Control, no. April 2013, pp. 14441463, 2013.

[11] G. P. Falconi, V. A. Marvakov, and F. Holzapfel, Fault tolerant control for a hexarotor system using Incremental Backstepping, 2016 IEEE Conf. Control Appl. CCA 2016, no. 1, pp. 237242, 2016.
[12] C.H. Lee, B.-E. Jun, and J.-I. Lee, Connections Between Linear and Nonlinear Missile Autopilots via Three-Loop Topology, J. Guid. Control. Dyn., vol. 39, no. 6, pp. 14241430, 2016.

[13] H. K. Khalil, Nonlinear System, Prentice-Hall, Upper Saddle River NJ, 1996.

[14] J. E. Slotin and W. Li, Applied Nonlinear Control, Prentice-Hall, Upper Saddle River, 1991

[15] M. Krstic, I. Kanellakopoulos and P. Kokotovic, Nonlinear and Adaptive Control, John Wiley and Sons, Inc., 1995

[16] B. S. Kim, Y. D. Kim, H. C. Bang, M. J. Tahk, and S. K. Hong, Flight Dynamics and Control, Kyung Moon Sa, 2004

[17] F. Golnaraghi, and B. C. Kuo, Automatic Control Systems, Wiley, 2009 
2018-10-29

\title{
Understandings of the incremental
} backstepping control through theoretical analysis under the model uncertainties

\author{
Jeon, Byoung-Ju
}

IEEE

Byoung ju Jeon, Minguk Seo, Hyo-sang Shin and Antonios Tsourdos. Understandings of the incremental backstepping control through theoretical analysis under the model uncertainties. Proceedings of the 2018 IEEE Conference on Control Technology and Applications (CCTA), 21-24 August, Copenhagen, Denmark.

https://doi.org/10.1109/CCTA.2018.8511479

Downloaded from Cranfield Library Services E-Repository 